University of Wollongong

Research Online

Faculty of Engineering and Information

Faculty of Engineering and Information

Sciences - Papers: Part A

Sciences

$1-1-2015$

\title{
Toward real-time multi-criteria decision making for bus service reliability optimization
}

Vu The Tran

Danang University of Science and Technology, vtt921@uowmail.edu.au

Peter Eklund

IT University of Copenhagen, peklund@uow.edu.au

Christopher Cook

University of Wollongong, chris_cook@uow.edu.au

Follow this and additional works at: https://ro.uow.edu.au/eispapers

Part of the Engineering Commons, and the Science and Technology Studies Commons

Research Online is the open access institutional repository for the University of Wollongong. For further information contact the UOW Library: research-pubs@uow.edu.au 


\title{
Toward real-time multi-criteria decision making for bus service reliability optimization
}

\author{
Abstract \\ This paper addresses issues associated with the real-time control of public transit operations to minimize \\ passenger wait time: namely vehicle headway, maintenance of passenger comfort, and reducing the \\ impact of control strategies. The randomness of passenger arrivals at bus stops and external factors \\ (such as traffic congestion and bad weather) in high frequency transit operations often cause irregular \\ headway that can result in decreased service reliability. The approach proposed in this paper, which has \\ the capability of handling the uncertainty of transit operations based on Multi-objective evolutionary \\ algorithm using a dynamic Bayesian network, applies preventive strategies to forestall bus unreliability \\ and, where unreliability is evident, restore reliability using corrective strategies. "Holding", "expressing", \\ "shortturning" and "deadheading" are the corrective strategies considered in this paper.

\section{Disciplines} \\ Engineering | Science and Technology Studies

\section{Publication Details} \\ Tran, V. The., Eklund, P. \& Cook, C. (2015). Toward real-time multi-criteria decision making for bus service \\ reliability optimization. In F. Esposito, O. Pivert, M. Hacid, Z. W. Ras \& S. Ferilli (Eds.), Proceedings of the \\ 22nd International Symposium on Methodologies for Intelligent Systems: Foundations of Intelligent \\ Systems (ISMIS 2015) (pp. 371-378). Switzerland: Springer.
}




\title{
Toward real-time multi-criteria decision making for bus service reliability optimization
}

\author{
Vu The Tran $(\bowtie)^{1}$, Peter Eklund ${ }^{2}$, and Chris Cook $^{3}$ \\ 1 Danang University of Science and Technology, Vietnam \\ ttvu@dut.udn.vn, \\ 2 IT University of Copenhagen, Denmark \\ petw@itu.dk \\ 3 University of Wollongong, Australia \\ ccook@@uow.edu.au
}

\begin{abstract}
This paper addresses issues associated with the real-time control of public transit operations to minimize passenger wait time: namely vehicle headway, maintenance of passenger comfort, and reducing the impact of control strategies. The randomness of passenger arrivals at bus stops and external factors (such as traffic congestion and bad weather) in high frequency transit operations often cause irregular headway that can result in decreased service reliability. The approach proposed in this paper, which has the capability of handling the uncertainty of transit operations based on Multi-objective evolutionary algorithm using a dynamic Bayesian network, applies preventive strategies to forestall bus unreliability and, where unreliability is evident, restore reliability using corrective strategies. "Holding", "expressing", "shortturning" and "deadheading" are the corrective strategies considered in this paper.
\end{abstract}

Keywords: Bayesian network, multi-objective optimization, public transit, transit service reliability, transit modeling and simulation, control strategies

\section{Introduction}

Measuring and reducing unreliability in a bus service is the focus of this paper. Unreliability affects passengers because it causes them to wait longer. Particularly on high frequency bus routes headway regularity is important to passengers because of its impact on waiting time and overcrowding. Overcrowding is a key to passengers because it impacts their comfort in a direct way and headway irregularity compounds operations because it slows boarding and alighting.

Passenger numbers are also important in transport planning because this measures network efficiency. For transit services with short headways, passengers can be assumed to arrive (more or less) randomly, namely independently of the schedule. Headway variability causes passengers to perceive that a service is unreliable, especially when "bunching" of buses occurs (clustering of the buses within a short distance of one another). 
The transit industry has (so far) lacked a measure of service reliability in terms of its impact on customers because traditional metrics do not express how much reliability impacts on passengers' perceptions. In this paper, service reliability is measured based on passenger wait time, comfort and bus headway [5].

In order to minimize unreliability, it is important to identify its possible causes in bus operations. Prevention strategies focus on reducing the variability of vehicle running and dwelling times, while corrective strategies focus on reducing negative impacts to passengers. Passenger costs, operation costs and implementation feasibility are used to evaluate corrective strategies. The most common corrective strategies are reviewed in this section: namely "holding", "expressing", "short-turning" and "deadheading" [8].

Corrective strategies, using headway and schedule optimization with bus location tracked in real-time is addressed by Dessouky et al. [4], Chen and Chen [2], $\mathrm{Yu}$ et al. [9], Daganzo and Pilachowski [3] and Bartholdi et al. [1]. These approaches develop real-time corrective strategies by coordinating buses along their route.

Among the corrective strategies, "expressing", "short-turning" and "deadheading" all involve station skipping but using varying strategies. "Expressing" involves sending a bus to a stop further downstream and skipping (not servicing) some, or all, intermediate stops. The objective of this strategy may be either to increase the headway between buses (separating bunched buses ) or to close a service gap further downstream, both in an attempt to balance headways and improve service past the end of the express segment [8].

Previous studies do not provide methods that have the ability to handle uncertainty in transit operations arising from within the transit environment and via the randomness of passenger arrivals. They also lack any mechanism that supports decision making for bus operations on route and at the bus stop simultaneously. This paper focuses on an approach for real-time multi-criteria decision-making based on dynamic Bayesian networks. These have the ability to handle uncertainty, reason about current states, and predict future states in cooperation with multi-objective optimization at each time slice in order to find appropriate strategies that maintain bus service reliability. The bus service reliability in our work takes into account passenger wait time, headway adherence, in-vehicle time, and passenger comfort, which are combined via Pareto comparisons in the fitness assignment processes.

The remainder of this paper is organized as follows: our proposed methodology for real-time decision making is presented in Section 2; simulation and results are reported and discussed in Section 3, with conclusions presented in Section 4.

\section{Proposed methodology}

Figure 1 shows the proposed methodology for controlling bus operations. In the real-time mode, a supervisor will receive evaluations of a current scenario including real-time travel demand, transit demand, bus network and assignment 


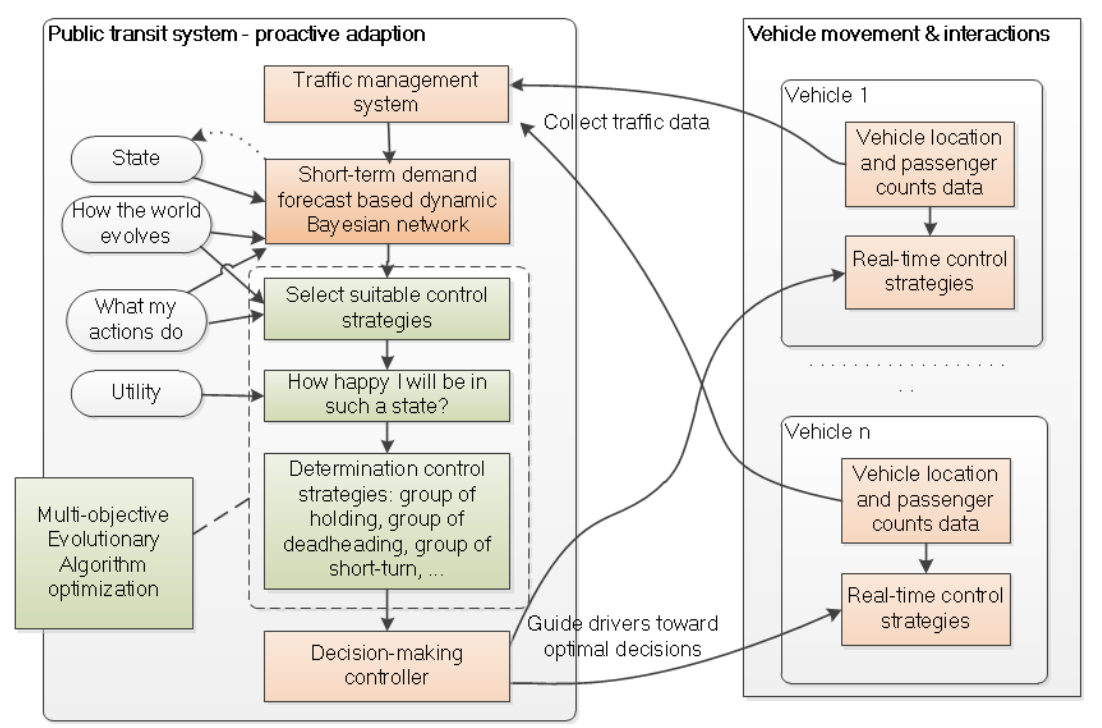

Fig. 1. Contextual Framework for bus operation control.

data. This is then used to give optimal proactive adaptation, including guidance for drivers leading to the goal of optimizing the bus network operations. The intention is to find strategies to guide drivers towards optimizing the overall bus network, not strategies solely for optimizing individual bus usage.

Real-time passenger demand and bus operation data are assumed to be collected from automatic passenger counting (APC) and automatic vehicle location (AVL) systems.

There are two main mechanisms in our control methodology: (1) state reasoning and demand prediction model, (2) multi-criteria decision making.

1. provides a mechanism that allows reasoning about current states and prediction about future states of bus operation based on a dynamic Bayesian network. This provides adequate information for (2) to make in-time and appropriate decision making.

2. provides a mechanism that allows suitable rational decision making for bus drivers on the route, namely preventive strategies, and at the bus stop, namely corrective strategies.

The details of these two mechanisms are described in the following two sub sections. 


\subsection{State reasoning and demand prediction model}

Rational decision-making in the context of this paper depends upon "both the relative importance of various goals and the likelihood that, and degree to which, they will be achieved" [7]. Probability offers a means of summarizing the uncertainty that originates from "laziness" and "ignorance". "Laziness" here means there is too much work in listing the complete set of antecedents and consequents needed to ensure an exception-less ruleset. The term "ignorance" splits in meaning between theoretical and practical. In theoretical terms "ignorance" here means there maybe no complete theory so the point at which a complete coverage of rules for the problem domain can never be adequately determined. In terms of practical "ignorance", even though all the rules are known, there is uncertainty about specific circumstances because not all the necessary deterministic tests have been (or can be) run [7]. Decision-making Bayesian networks have the ability to handle these types of uncertainty.

In order to monitor the state of the system over a specific period of time, a dynamic Bayesian network model [7][6] is proposed. Fig. 2 shows a dynamic Bayesian network model with $t$ time slices for a bus network based on the static network, which includes three types of nodes: chance nodes (ovals), decision nodes and utility nodes (diamonds). The set of variables of chance node is $\mathcal{X}_{\Gamma}=$ speed $\mathcal{V}_{i}$, position $\mathcal{X}_{i}$, number of passengers alighting $\mathcal{A}_{i, k}$ of vehicle $i$ at stop $k$, number of passengers boarding $\mathcal{B}_{i, k}$, running time $\mathcal{R}_{i, k}$, dwell time $\mathcal{D}_{i, k}$, in-vehicle load $\mathcal{L}_{i, k}$, headway adherence $\mathcal{H}_{\text {adherence, }}$, passenger wait time $\mathcal{T}_{\text {wait }}$, action impact $\mathcal{T}_{\text {impact }}$, passenger comfort $\left.\xi_{\text {comfort }}\right\}$. Action of decision node has state $\mathcal{X}_{D}=\left\{\right.$ no action, preventive control $p x_{i, k}$, holding $h x_{i, k}$, expressing $e x_{i, k}$, short-turning $s x_{i, k}$, deadheading $\left.d x_{i, k}\right\}$. The utility node represents the expected utility associated with each action.

Each time step models the state of the bus network at a specific point in time; the dashed lines present the separation of the model into time slices. In Figure 2 , smoothing is the process of querying about the state of the bus network at a previous time step from the current time, while filtering is the process of querying and predicting the state of the system from the current time to future steps. The conditional probability distributions $P\left(\mathcal{V}_{i}^{t} \mid \mathcal{V}_{i}^{t-1}\right), P\left(\mathcal{X}_{i}^{t} \mid \mathcal{X}_{i}^{t-1}\right)$, $P\left(\mathcal{A}_{i}^{t} \mid \mathcal{A}_{i}^{t-1}\right), P\left(\mathcal{B}_{i}^{t} \mid \mathcal{B}_{i}^{t-1}\right) P\left(\mathcal{R}_{i, k}^{t} \mid \mathcal{R}_{i, k}^{t-1}\right), P\left(\mathcal{D}_{i, k}^{t} \mid \mathcal{D}_{i, k}^{t-1}\right)$ and $P\left(\mathcal{L}_{i, k}^{t} \mid \mathcal{L}_{i, k}^{t-1}\right)$ are the relevant transition probability distributions. The state of the bus network at the current point in time will impact the state of the system in the future and be impacted by the state of the system in the past. The development of the bus network is specified by links between variables in different time-slices. In this paper, the interval between slices is assumed to be fixed. For monitoring bus network states, a practical interval is 5 minutes.

\subsection{Multi-criteria decision making}

Decision making for bus operations to provide service reliability in this paper is driven by a set $f$ of four objective functions $\left(f=f_{1}, f_{2}, f_{3}, f_{4}\right)$, where $f_{1}$ is passenger wait time, $f_{2}$ is headway adherence, $f_{3}$ is passenger comfort, and $f_{4}$ is 


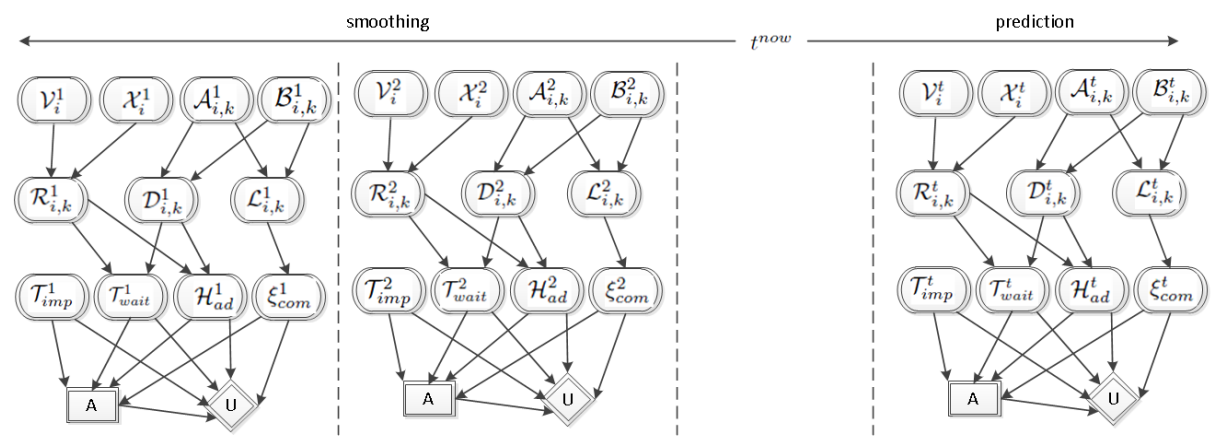

Fig. 2. Bus network time slices.

impact of control strategies. These functions are combined via Pareto comparisons in the fitness assignment processes.

$$
\begin{gathered}
f_{1}=\mathcal{T}_{\text {wait }}=\sum_{i=1}^{n} \sum_{k=1}^{m}\left(\frac{\lambda_{k}\left(\widetilde{\mathcal{A D}}_{i, k}-\mathcal{A D}_{i-1, k}\right)^{2}}{2}+\mathcal{P}_{i, k}\left(\widetilde{\mathcal{A D}}_{i, k}-\mathcal{A D}_{i-1, k}\right)\right) \\
f_{2}=\mathcal{H}_{\text {adherence }}=\sum_{i=1}^{n} \sum_{k=1}^{m}\left(\widetilde{\mathcal{H}}_{i, k}-\mathcal{S H}\right)^{2} \\
f_{3}=\xi_{\text {comfort }}=\sum_{i=1}^{n} \sum_{k=1}^{m} \frac{\widetilde{\mathcal{L}}_{i, k}}{\mathcal{L}_{m a x}} \\
f_{4}=\mathcal{T}_{\text {impact }}=\sum_{i=1}^{n} \sum_{k=1}^{m} \widetilde{\mathcal{L}}_{i, k} \times h x_{i, k}+\left(\text { ex }_{i, k}+d x_{i, k}+s x_{i, k}\right) \times \widetilde{\mathcal{H}}_{i, k} \times \widetilde{\mathcal{P D}}_{i, k}
\end{gathered}
$$

Handling multi-objective problems, namely multi-criteria optimization, can be described as a process of finding the vector of decision variables $\boldsymbol{x}^{*}=\left(x_{1}^{*}, x_{2}^{*}, \ldots, x_{n}^{*}\right)$, where $n$ is number of buses and $x_{i}^{*} \in\{h x, e x, d x, s x, p x\}$ is the control strategy applying for bus $i$ at decision time, which minimizes the vector function,

$$
\min \boldsymbol{f}(\boldsymbol{x})=\left(w_{1} \times f_{1}(\boldsymbol{x})+w_{2} \times f_{2}(\boldsymbol{x}), f_{3}(\boldsymbol{x}), f_{4}(\boldsymbol{x})\right)
$$

where $\boldsymbol{x}=\left(x_{1}, x_{2}, \ldots, x_{n}\right) \in \Omega \in R^{n}$ is called the decision variable, the set $\Omega$ is called the feasible region. Figure 3 depicts control sequence encoding of $n$ buses. Each bus selects a control from the list. The solution will balance the optimization of an individual bus and the whole bus network.

In this paper, objective functions $f_{1}$ and $f_{2}$ are considered to be optimizeable simultaneously. This can mean that improvement of one can lead to an improvement of the other. $f_{1}$ and $f_{2}$, hence, use a weighted-sum approach. $f_{4}$ 


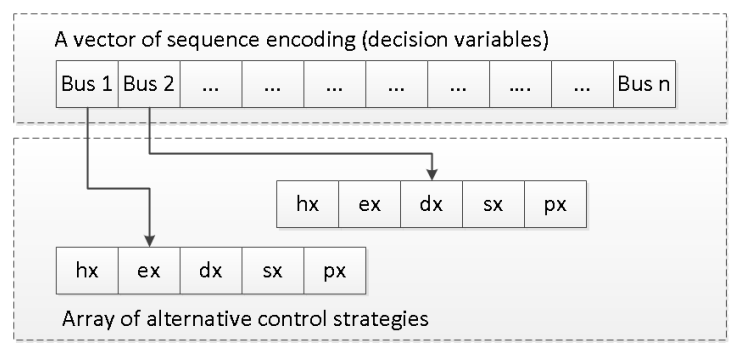

Fig. 3. Control sequence encoding

is considered to conflict with $f_{1}$ and $f_{2}$ while $f_{3}$ is independent so it does not influence any other objective function.

Station reasoning and demand prediction model in Section 2.1 provides information about current and predicted bus service reliability, which are used to decide whether multi-objective optimization process should be run. If service unreliability is predicted, the optimization algorithms will advise the decision making for buses at selected bus stops to restore the reliability.

A multi-Objective Evolutionary Algorithm (MOEA) is proposed for handling multi-objective problems in this paper. Deterministic algorithms are most often used if a clear relationship exists between the characteristics of the possible solutions. If the relation between a solution candidate and its "fitness" are not so obvious, as in the case of transit operation, probabilistic algorithms come into play.

\section{Simulation and results}

\subsection{Simulation}

A case study of bus operations on the Gwynneville-Keiraville bus route in the regional city of Wollongong, Australia (population 300,000) is used to demonstrate and test the simulator. The simulator deals with a single time period, namely the peak period from 16:34 to $22: 32$ on weekdays.

\subsection{Results and analysis}

The non-dominated solutions of the three objectives: $f_{1}, f_{2}, f_{3}$ obtained from 100 iterations are shown in Fig. 4 by blue-colored points. Fig. 4 shows the trade-off between passenger wait time and action impact and trade-off between passenger wait time and passenger comfort. Square points represent evolutionary algorithm solutions, circle points represent pseudo-optimal pareto front.

After generating a Pareto optimal configuration with the set of good solutions, several key performance indicators are calculated for each solution. Decision makers can also choose any non-dominated solution from an experimental run based on their preference weight vectors. 

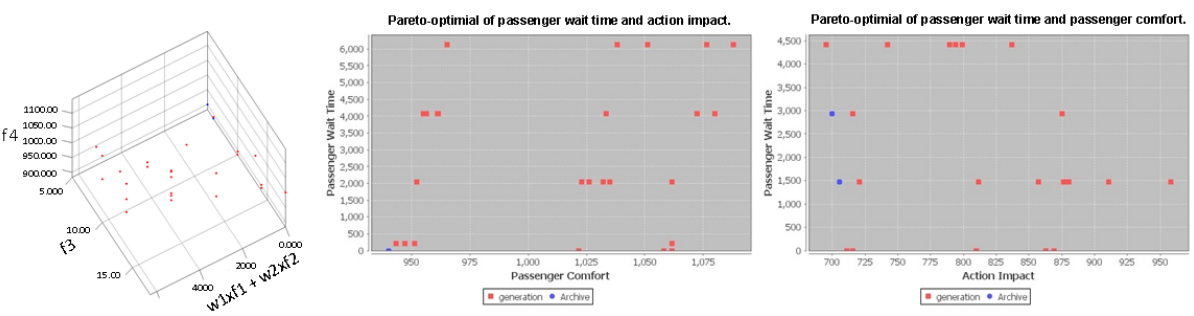

Fig. 4. Pareto-optimial.

Performance parameters used in this paper are used to address transit service reliability from the perspective of passengers. For short-headway services, the variability of headways is the main route-based measure for evaluating transit reliability. An effective control strategy improves service reliability by reducing headway variability, which in turn results in shorter passenger waiting times. Charts 1 and 2 of Fig. 5 presents space-time headway adherence before and after applying control strategies for the peak hour $(16: 30-23: 00)$. There is more bunching before applying control strategies.
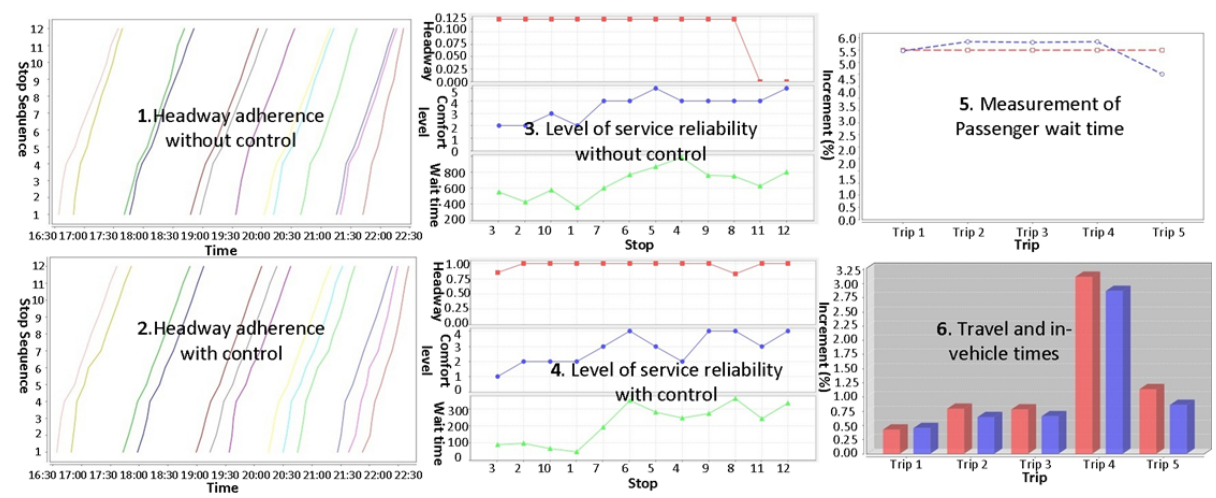

Fig. 5. Measure of Efficiency

Another performance route-based measure is passenger wait time. Charts 3 and 4 of Fig. 5 shows passenger wait time compared with expected wait time and the effect of control strategies on travel and in-vehicle times.

Control strategies may cause delays to on-board passengers and longer travel times that may result in higher fleet costs. However, improved regularity of headways can reduce the in-vehicle time of the passengers at the subsequent stops. In addition, passenger waiting time at bus stops can in practice be considered more important than passenger in-vehicle waiting time.

Bus reliability at the stop level is considered from a passengerś point of view, which can be used to enhance reliability from a passengerś perspective. Line 
charts in Fig. 5 used to measure stop-level bus reliability with (and without) employing control strategies.

The results in charts 5 and 6 of Fig. 5 indicate that there was low service reliability for the bus transit network before applying the control strategies. Applying control strategies helps to dramatically improve service reliability.

\section{Conclusion}

Our Multi-objective Evolutionary algorithm based dynamic Bayesian networks approach provides the ability to reason and predict bus service reliability network as well as to handle multi-criteria decision making to control real-time information. It is able to handle uncertainty which, when presented through variables based on probability and its dynamic choosing action, yields the highest expected utility. Another advantage of our approach is that it considers headway adherence, running time, dwell time and decision-making as continuous values. The effect is that the algorithm is more flexible in decision-making compared to existing transit control methods.

A simulation-based evaluation enables us to verify the efficiency of our approach. The simulation examined performance and level-of-service by capturing the interactions between transit operations and passenger demand. Paretooptimal analysis is used to measure efficiency of a multi-objective evolutionary algorithm. Route and stop level analysis for transit service reliability improves passenger decision-making processes and enhances daily route service management by the transit agents.

\section{References}

1. J.J. Bartholdi and D.D. Eisenstein. A self-coördinating bus route to resist bus bunching. Transportation Research Part B: Methodological, 46(4):481-491, 2012.

2. W.Y. Chen and Z.Y. Chen. A simulation model for transit service unreliability prevention based on avl-apc data (pdf). 2009.

3. C.F. Daganzo and J. Pilachowski. Reducing bunching with bus-to-bus cooperation. Transportation Research Part B: Methodological, 45(1):267-277, 2011.

4. M. Dessouky, R. Hall, L. Zhang, and A. Singh. Real-time control of buses for schedule coordination at a terminal. Transportation Research Part A: Policy and Practice, 37(2):145-164, 2003.

5. P.G. Furth. Using archived AVL-APC data to improve transit performance and management, volume 113. Transportation Research Board National Research, 2006.

6. U.B. Kjaerulff and A.L. Madsen. Bayesian networks and influence diagrams: a guide to construction and analysis. Springer Verlag, 2007.

7. S.J. Russell and P. Norvig. Artificial intelligence: a modern approach. Prentice hall, 2010.

8. N.H.M. Wilson, L.C. Cham, et al. Understanding bus service reliability: a practical framework using AVL/APC data. PhD thesis, Massachusetts Institute of Technology, 2006.

9. B. Yu, Z. Yang, X. Sun, B. Yao, Q. Zeng, and E. Jeppesen. Parallel genetic algorithm in bus route headway optimization. Applied Soft Computing, 2011. 